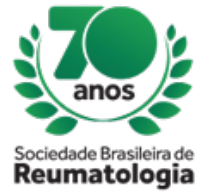

\title{
EPIDEMIOLOGICAL ASPECTS AND THE TREATMENT OF OSTEOPOROSIS IN VERY ELDERLY PATIENTS
}

Luiza Trincado (HSPE - IAMSPE, São Paulo, SP, Brasil), Elisa Pereira Vicentini (HSPE - IAMSPE, São Paulo SP, Brasil), Estevão da Costa Santana (HSPE - IAMSPE, São Paulo, SP, Brasil), Ana Clara Ribeiro (HSPE -

IAMSPE, São Paulo, SP, Brasil), Ana Paula Dauricio de Mattos (HSPE - IAMSPE, São Paulo , SP, Brasil),

Natália Carneiro dos Santos (HSPE - IAMSPE, São Paulo, SP, Brasil), Elaine de Azevedo (HSPE - IAMSPE, São Paulo, SP, Brasil), Rina Dalva Neubarth Giorgi (HSPE - IAMSPE, São Paulo , SP, Brasil)

\section{BACKGROUND}

The incidence of osteoporotic fractures increases with age. Age is one of the major determinants, not only for the absolute risk of fracture, but also for the type of osteoporotic fracture. Osteoporosis (OP) in very old individuals is underdiagnosed and under-treated. There is a lack of data related to the epidemiological aspects of this subgroup and conclusive evidences regarding the efficacy of the available treatments. In this paper we will discuss some of these data observed in patients over 85 years of age, with a diagnosis of $\mathrm{OP}$, followed in a referenced service.

\section{MATERIALS AND METHODS}

A review of 91 medical records, belonging to very old patients with a mean age of 89.5 years, who were followed up at the osteoporosis clinic, and tabulated the main characteristics, such as sex, ethnicity, time of disease, pharmacological treatment (previous and current), presence and site of fracture by fragility, among other information.

\section{RESULTS}

As to gender and ethnicity, 81 were female and 10 were males, 73 were of Caucasian ethnicity, while 18 were of other ethnicities. The mean follow-up time for osteoporosis is 10.5 years.

Of the total, 86 patients (94.5\%) are or have already taken at least one medication of the bisphosphonate class. The current medication was divided into $44 \%$ of patients taking zoledronic acid, $34 \%$ taking denosumab, $6 \%$ taking teriparatide, 3.3\% using risedronate, 2,2\% taking raloxifene, and 9.9\% \% on "drug holiday".

Regarding the presence of fragility fracture, 72 patients ( $80 \%$ of the cases) had one or more fractures. The sites were: $29 \%$ in the lumbar spine, $19 \%$ in the dorsal spine, $16 \%$ in the femur, $16 \%$ on the wrist, $8.3 \%$ in rib, $2.7 \%$ in clavicle and $12,5 \%$ in other places. Five patients had more than one fracture concomitantly.

Among the factors analyzed, the one that presented the greatest correlation with the risk of fracture was the family history of osteoporosis. In the population with a family history of fractured osteoporosis, everyone had at least one fragility fracture.

\section{CONCLUSION}

With aging, the incidence of age-related conditions, especially OP, increases. Fractured elderly usually have several comorbidities and should be considered fragile. This fragility reflects in post-fracture outcomes such as increased mortality, functional decline and loss of quality of life. Special attention should be given to data easily collected in the interview such as the presence of family history of osteoporosis with fracture, which in our population was the most striking finding regarding the risk of fracture. 